\title{
Decision-Related Activity in Macaque V2 for Fine Disparity Discrimination Is Not Compatible with Optimal Linear Readout
}

\author{
Stephane Clery, ${ }^{1}{ }^{\circledR}$ Bruce G. Cumming, ${ }^{2}$ and ${ }^{\circledR}$ Hendrikje Nienborg ${ }^{1}$ \\ ${ }^{1}$ University of Tuebingen, Werner Reichardt Centre for Integrative Neuroscience, 72076 Tuebingen, Germany, and ${ }^{2}$ Laboratory of Sensorimotor Research, \\ National Eye Institute, National Institutes of Health, Bethesda, Maryland 20892
}

Fine judgments of stereoscopic depth rely mainly on relative judgments of depth (relative binocular disparity) between objects, rather than judgments of the distance to where the eyes are fixating (absolute disparity). In macaques, visual area V2 is the earliest site in the visual processing hierarchy for which neurons selective for relative disparity have been observed (Thomas et al., 2002). Here, we found that, in macaques trained to perform a fine disparity discrimination task, disparity-selective neurons in V2 were highly selective for the task, and their activity correlated with the animals' perceptual decisions (unexplained by the stimulus). This may partially explain similar correlations reported in downstream areas. Although compatible with a perceptual role of these neurons for the task, the interpretation of such decision-related activity is complicated by the effects of interneuronal "noise" correlations between sensory neurons. Recent work has developed simple predictions to differentiate decoding schemes (Pitkow et al., 2015) without needing measures of noise correlations, and found that data from early sensory areas were compatible with optimal linear readout of populations with informationlimiting correlations. In contrast, our data here deviated significantly from these predictions. We additionally tested this prediction for previously reported results of decision-related activity in V2 for a related task, coarse disparity discrimination (Nienborg and Cumming, 2006), thought to rely on absolute disparity. Although these data followed the predicted pattern, they violated the prediction quantitatively. This suggests that optimal linear decoding of sensory signals is not generally a good predictor of behavior in simple perceptual tasks.

Key words: choice probability; discrimination; disparity; nonhuman primate; readout; V2

Significance Statement

Activity in sensory neurons that correlates with an animal's decision is widely believed to provide insights into how the brain uses information from sensory neurons. Recent theoretical work developed simple predictions to differentiate decoding schemes, and found support for optimal linear readout of early sensory populations with information-limiting correlations. Here, we observed decision-related activity for neurons in visual area V2 of macaques performing fine disparity discrimination, as yet the earliest site for this task. These findings, and previously reported results from V2 in a different task, deviated from the predictions for optimal linear readout of a population with information-limiting correlations. Our results suggest that optimal linear decoding of early sensory information is not a general decoding strategy used by the brain.

\section{Introduction}

In humans (Westheimer, 1979; McKee and Levi, 1987) and monkeys (Prince et al., 2000), judgments of small differences in stereoscopic depth rely on relative binocular disparity (i.e., the

Received July 31, 2016; revised Nov. 20, 2016; accepted Nov. 29, 2016.

Author contributions: B.G.C. and H.N. designed research; H.N. performed research; S.C. and H.N. analyzed data; S.C., B.G.C., and H.N. wrote the paper.

This work was supported by European Research Council (Project NEUROOPTOGEN) Starting Independent Researcher Grant to H.N. and the National Institutes of Health, National Eye Institute Intramural Research Program. We thank Denise Parker for excellent animal care.

The authors declare no competing financial interests. comparison of differences in nearby disparities in the stimulus). The first stage in the primate cortex that contains a subgroup of neurons selective for relative disparity is visual area $\mathrm{V} 2$ (Thomas et al., 2002; Neri et al., 2004). Although we have previously shown that the activity of disparity-selective neurons in V2 is correlated with a macaque monkey's perceptual decision in a "coarse" dis-

Correspondence should be addressed to Dr. Hendrikje Nienborg, University of Tuebingen, Werner Reichardt Centre for Integrative Neuroscience, Otfried Mueller Strasse 25, 72076 Tuebingen, Germany. E-mail: hendrikje.nienborg@cin.uni-tuebingen.de.

DOI:10.1523/JNEUROSCI.2445-16.2016

Copyright $\odot 2017$ the authors $\quad 0270-6474 / 17 / 370715-11 \$ 15.00 / 0$ 


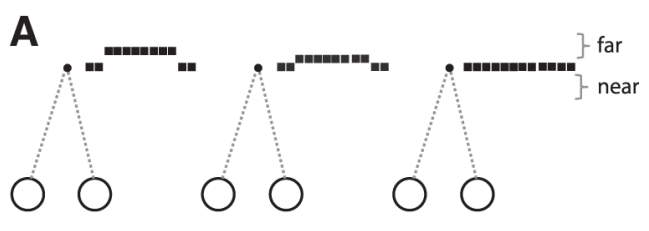

B

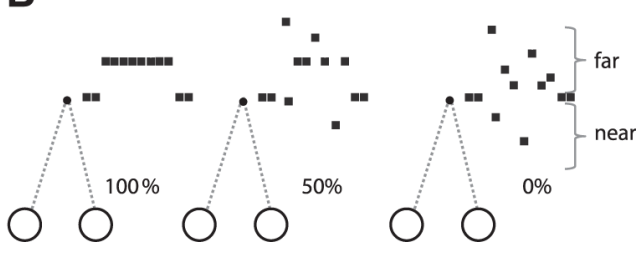

Figure 1. The fine and coarse disparity discrimination tasks. A, Stimulus illustration of the fine disparity discrimination task with an example far stimulus. The surrounding annulus is at the disparity of the fixation plane $\left(0^{\circ}\right)$. The disparity difference between the central region and the surround is smaller in the middle than the left panel, which increases the discrimination difficulty. The stimulus on the right corresponds to the $0 \%$ signal condition for which the disparity of the center is the same as for the surrounding annulus. $\boldsymbol{B}$, Schematic of the coarse disparity discrimination task. The signal disparity is far and well above threshold. For the $50 \%$ signal stimulus, $50 \%$ of the dots in the RDS are replaced by noise dots. For the $0 \%$ signal stimulus, the central patch of the stimulus consists only of noise dots.

parity discrimination task (Fig. 1B) (Nienborg and Cumming, 2006, 2007, 2009), no one has examined this correlation for "fine" tasks that depend on relative disparity (Fig. 1A). In other extrastriate areas, such decision-related activity has been reported for neurons in the inferior-temporal cortex, area IT (Uka et al., 2005) and V4 (Shiozaki et al., 2012) that also has neurons selective for relative disparity (Umeda et al., 2007). Conversely, this correlation is absent in area MT (DeAngelis, 2009), where MT neurons are not selective for relative disparity when tested with the same stimulus (Uka and DeAngelis, 2006). Other stimulus configurations do reveal selectivity for relative disparity in MT (Krug and Parker, 2011). Here we examine the possibility that some of this decision-related activity in downstream areas may be inherited from V2 neurons. Given the heterogeneous selectivity for relative disparity in V2 it is possible that V4 and IT inherit decision-related activity from a subset of $\mathrm{V} 2$ neurons that are selective for relative disparity, whereas MT inherits decisionrelated activity from the V2 neurons that are not selective for relative disparity. To address this question, we recorded the activity of disparity-selective neurons in V2 of 2 macaque monkeys while they performed a fine disparity discrimination task, and examined whether this was related to their selectivity for relative disparity. We also compare these results with the decision-related activity we previously reported for coarse disparity discrimination in V2 in the same animals (Nienborg and Cumming, 2006, 2007, 2009).

Comparing decision-related activity at different processing levels and for different tasks potentially offers additional insights into the origins of decision-related activity in sensory neurons, and into how the brain is reading out the sensory information. For many discrimination tasks, decision-related activity depends on the tuning preferences of the neuron and increases with a neuron's sensitivity for the task (Celebrini and Newsome, 1994; Britten et al., 1996; Romo et al., 2002; Uka and DeAngelis, 2004; Purushothaman and Bradley, 2005; Gu et al., 2008; Liu et al., 2013a, b; Nienborg and Cumming, 2014). This suggests that the correlation reflects a specific link between task-relevant sensory neurons and the animal's perceptual choice. Nonetheless, the interpretation of such decision-related activity has proven diffi- cult (Shadlen et al., 1996; Krug, 2004; Nienborg and Cumming, 2010; Nienborg et al., 2012; Haefner et al., 2013; Crapse and Basso, 2015; Nienborg and Roelfsema, 2015; Smolyanskaya et al., 2015; Cumming and Nienborg, 2016). In particular, it is complicated (Shadlen et al., 1996; Cohen and Newsome, 2009; Haefner et al., 2013) by the presence of interneuronal correlations ("noise-correlations"; for review, see Cohen and Kohn, 2011), typically observed between pairs of sensory neurons. A recent study (Pitkow et al., 2015), building on analytical work (Haefner et al., 2013; Moreno-Bote et al., 2014), identified a distinctive property of networks in which choice is determined by an optimal linear readout of sensory neurons with information-limiting correlations, regardless of other details of those correlations. This property, a specific relationship between decision-related activity, neuronal performance, and behavior, was compatible with data reported in dorsal medial superior temporal area for a heading discrimination task. If this relationship holds in V2 for either disparity task, it would suggest that decision-related activity downstream is simply inherited from V2. (If the downstream area receives additional information and is also optimally decoded, the behavior would have to be better than an optimal decoder of $\mathrm{V} 2$ alone.) If the predicted relationship does not hold in V2, it raises questions about the origin of the decision-related activity downstream. For example, larger than predicted choice probabilities (CPs) imply a choicerelated signal that is not derived from the limiting noise (Pitkow et al., 2015). If such a signal is present in V2, it is presumably passed downstream.

\section{Materials and Methods}

Animals. All procedures reported in this study were performed in compliance with the Public Health Service policy on humane care and use of laboratory animals and all protocols were approved by the National Eye Institute Animal Care and Use Committee. We studied the neural activity of disparity-selective neurons from visual area V2 in two male macaque monkeys (Macaca mulatta). Before recording scleral search coils (Judge et al., 1980), a headpost and a recording chamber over the operculum of V1 were implanted under general anesthesia (for a more detailed description, see Cumming and Parker, 1999).

Recording. Recording of neural activity was done while the animals were performing a disparity discrimination task. We also recorded eye positions for each eye (CNC Engineering). The procedure used for recording was described previously (Cumming and Parker, 1999; Nienborg et al., 2004, 2005). Only activity from isolated units is reported in this study. Spike sorting was performed offline using custom-written software.

Before starting the behavioral experiment, we determined the size and position of the receptive field (Read and Cumming, 2003) and the disparity tuning of the recorded unit while the animal was required to maintain fixation within 1 degree of a 0.1 degree fixation point. Although we do not have histological data, we find a substantial correlation between disparity selectivity and direction selectivity in V2 (H.N. and B.G.C., unpublished observations). Although it therefore seems likely that the majority of our disparity-selective neurons come from thick stripes (e.g., Chen et al., 2008; Lu et al., 2010), it is possible that a substantial number of units were also recorded outside the thick stripes.

Task. Once we characterized the disparity tuning properties of a unit, we recorded its extracellular activity during a fine disparity discrimination task. The animals' task was to discriminate whether the central disc was protruding ("near") or receding ("far") relative to a surrounding annulus (typically 1 degree wide). In this task, the center disparity was varied in small increments around the surround disparity. In each session, the disparity of the surrounding disk was kept constant while the disparity of the center disk was varied randomly between trials. We report new data for the fine disparity discrimination task and compare these with previously reported data obtained for the coarse disparity discrimination task (Nienborg and Cumming, 2006, 2007, 2009). 
The monkeys initiated trials by fixating a fixation point at the center of the display. The stimulus was then presented for $2 \mathrm{~s}$ while the monkeys were required to maintain fixation within 0.5 degrees of the fixation point. After stimulus presentation, two choice targets appeared and the animals responded by making a saccade either upward (indicating a "far" choice) or downward ("near" choice). If correct, the monkeys received a liquid reward.

Whenever possible, data for both the fine and the coarse disparity discrimination task were collected for the same unit in consecutive blocks during the same recording session.

Stimuli. Stimuli were generated on a Silicon Graphics workstation using custom-written software. Stimuli were presented via a Wheatstone stereoscope configuration. This consisted of two Flexscan F980 monitors (EIZO) and a set of mirrors oriented at $45^{\circ}$, positioned at $1.5 \mathrm{~cm}$ from the eyes. The viewing distance was $89 \mathrm{~cm}$. For each monitor, the mean luminance on screen was $42 \mathrm{~cd} / \mathrm{m}^{2}$, the maximum contrast was $99 \%$, and the frame rate was $96 \mathrm{~Hz}$.

The stimuli were dynamic random dot stereograms (RDS, 50\% black, $50 \%$ white, typical dot density $40 \%$ and $0.09 \times 0.09$ degrees dot size). The RDS consisted of a center disk and a surrounding annulus ("background"). Its size (mean size of the center: 3.8 degrees; mean width of the annulus: 1.3 degrees) and position were fit to the receptive field (mean eccentricity: 4.86 degrees) of the recorded unit.

For the fine disparity discrimination task, we used dynamic RDS at $100 \%$ binocular correlation. For each recorded unit, we tailored the surround disparity to the tuning preference of the neuron such that the neuron's disparity tuning had a steep slope in the vicinity of the surround disparity. The surround disparity remained fixed within each session. The median value of the surround disparity was 0 degrees (range: -0.5 to 0.3 ). For a surround disparity of 0 , a typical range of disparities (in degrees) for the central disc was as follows: $-0.05,-0.025,-0.0125,0$, $0.0125,0.025,0.05$. On trials for which the center disparity was identical to the surround disparity ( $0 \%$ signal trials), the monkeys were rewarded on $50 \%$ of the trials chosen randomly.

Whenever possible, we also measured the responses of the same unit while the monkeys performed a coarse disparity discrimination task. The stimuli for the coarse disparity discrimination task are described in detail in Nienborg and Cumming $(2006,2009)$. Briefly, for each coarse disparity discrimination task, we chose one near and one far signal disparity both well above disparity detection threshold, which were chosen to be close to the preferred and null disparity of the recorded neuron. The surrounding annulus was at 0 degrees disparity, at $100 \%$ binocular correlation. We used noise to manipulate task difficulty in the coarse disparity discrimination task and varied the proportion of signal and noise between trials. For the comparison of CP in the coarse versus fine disparity discrimination task (see Fig. 7), we included data for which two versions of noise were used. In the first version, noise dots correspond to dots that are uncorrelated between the eyes (Nienborg and Cumming, 2006, 2007). On trials with $0 \%$ signal dots, all dots were uncorrelated and the monkeys were rewarded randomly on $50 \%$ of the trials. In the second version, all the dots of the center had the same disparity on each video frame (frame rate $196 \mathrm{~Hz}$ ) while this disparity value changed randomly from frame to frame. For the condition with no added signal ( $0 \%$ signal trials), the disparity on each frame was drawn at random from a uniform distribution of discrete, equally spaced disparities, symmetrical around 0 degree disparity, and encompassing the preferred and the null disparity of each neuron. For these trials, the monkeys were rewarded randomly on $50 \%$ of the trials. Disparity signal was introduced by increasing the probability of the signal disparity on each frame (for a detailed description, see Nienborg and Cumming, 2009).

Measurements of relative disparity tuning. To quantify tuning for relative or absolute disparity, we measured the responses to RDS stimuli for which we systematically varied the disparity of the center and the surrounding annulus (typically 7 center disparities, each presented at 7 different surround disparities, typically spanning 1 degree), presented in random order. Stimulus presentation lasted $450 \mathrm{~ms}$. The resulting 2D response map (center disparity $\times$ surround disparity) was fitted with a 2D Gabor function (see Fig. 5, insets), and selectivity for relative or absolute disparity summarized by the orientation of the 2D Gabor. Ver- tical orientation (90 degrees) indicates that the selectivity to the disparity in the center is independent of the disparity in the surround (absolute disparity tuning). An oblique orientation indicates that the neuron changes its center disparity selectivity depending on the disparity present in its surround. Only cells for which the 2D Gabor fits explained $>65 \%$ of the variance were included for this analysis. We determined whether the orientation of the 2D Gabor was significantly lower than 90 degrees by resampling (1000 resamples).

Computation of CP. CP quantifies the trial-by-trial correlation between an animal's choice and the spike count of a unit (Britten et al., 1996). We computed the CP using only $0 \%$ signal trials. The spike count over the whole $2 \mathrm{~s}$ stimulus presentation was used and spike counts were separated by choice. $\mathrm{CP}$ was computed as the area under the receiver operating characteristic (ROC) curve. A permutation test (Britten et al., 1996; Uka and DeAngelis, 2003) was used to assess whether a unit had significant $\mathrm{CP}$. For the comparison with $\mathrm{CP}$ in the coarse discrimination task (see Fig. 7), CP was computed in response to $0 \%$ signal stimuli. For data obtained for the version of the coarse disparity discrimination task used by Nienborg and Cumming (2009), CP was corrected for stimulusinduced variability.

Neurometric and psychophysical thresholds. Neuronal thresholds were calculated based on ROC analysis (applied to mean firing rates during the entire $2 \mathrm{~s}$ trial) using a "neuron-antineuron" formulation as described previously (Britten et al., 1996; Shiozaki et al., 2012). (This formulation assumes a theoretical "antineuron" with opposite disparity tuning, but otherwise identical responses to that of the actual neuron recorded.) The neurometric curves were then plotted as a function of the signed disparity signal (center disparity - surround disparity) relative to the preference of the neuron. For the psychophysical functions, the percentage of choices to a neuron's preferred disparity were plotted as a function of the signed disparity signal. Both the psychophysical and neurometric function were then fitted with a cumulative Gaussian via maximum likelihood. The SDs of the cumulative Gaussian functions were defined as the neurometric and psychometric threshold, respectively, and correspond to the $84 \%$ correct level. Only fits that explained $>65 \%$ of the variance were included. For the comparison with values for the coarse disparity discrimination task (see Fig. $8 B$ ), we used an extended dataset of previously published data (Nienborg and Cumming, 2006, 2007).

Inclusion criteria. We recorded from 111 single units (52 and 59 from Monkeys 1 and 2, respectively) and only included cells that were significantly tuned for disparity ( $p<0.01$ on a one-way ANOVA) and whose preferred disparity yielded a response of at least $5 \mathrm{spk} / \mathrm{s}$.

We further applied a number of behavioral criteria to our dataset. First, we excluded sessions for which the animals had a strong behavioral bias (choosing one target $>80 \%$ on $0 \%$ signal trials). Additionally, the animals had to perform the task at least $80 \%$ correctly, for at least one of the two easiest "near" and "far" disparity stimuli, respectively. Finally, only sessions for which the monkey made at least three near and far choices each for the $0 \%$ signal trials were included. These criteria were met by $n=84$ neurons (Monkey 1: $n=38$; Monkey 2: $n=46$ ). In Monkey 2, the mean vertical eye position differed systematically in a subset of sessions such that there was a significant negative correlation between the mean choice-dependent difference in eye position and $\mathrm{CP}$ $(r=-0.29, p=0.048)$. We therefore included an additional criterion to exclude sessions for which there were systematic differences in the mean vertical eye position associated with the two choices that exceeded 0.12 degrees. This criterion abolished the negative correlation between the mean choice-dependent difference in vertical eye position and CP in Monkey 2 $(r=0.009, p=0.96)$. The dataset for which we measured CPs therefore consists of 63 single units (Monkey 1: $n=37$; Monkey 2: $n=26$ ).

Choice correlations. To test predictions for an optimal linear readout of V2 in the presence of correlated noise (Pitkow et al., 2015), we converted our measured CPs into choice correlations (Pitkow et al., 2015) according to the following equation:

$$
\text { choice correlation }_{\text {measured }} \approx \frac{\pi}{\sqrt{2}}\left(\mathrm{CP}-\frac{1}{2}\right)
$$


This value was then compared with the choice-correlation value predicted for an optimal linear decoder in the presence of informationlimiting correlated noise (Pitkow et al., 2015) as follows:

$$
\text { choice correlation }_{\text {predicted opt }}=\frac{\text { psychophysical threshold }}{\text { neurometric threshold }}
$$

This prediction (Pitkow et al., 2015) was developed for neurometric thresholds computed by comparing the responses of one neuron to a fixed reference. We compute neurometric thresholds with the traditional "neuron-antineuron" method. The signal amplitude is therefore twice as large (neuron-antineutron compared with neuron-constant), but also has twice the variance. This produces neurometric thresholds that are smaller by $\sqrt{2}$ (for a fuller discussion how the "neuron-antineuron" formulation influences neurometric thresholds, see Prince et al., 2000). We therefore multiplied our neurometric thresholds by $\sqrt{2}$ before comparing them with choice correlations. To test this prediction for the coarse disparity discrimination task (see Fig. $8 D$ ), we used an extended dataset of previously published data (Nienborg and Cumming, 2006, 2007).

\section{Results}

We recorded the extracellular activity from disparity-selective neurons in V2 while 2 macaque monkeys performed a fine disparity discrimination task. The stimuli consisted of circular dynamic RDSs surrounded by a circular annulus and presented at $100 \%$ interocular correlation. For each session, the surround disparity was tailored to the disparity preferences of the neuron such that small changes around this disparity value corresponded to the steep slope of the neuron's disparity tuning curve (compare Fig. 2). The animals' task was to judge whether the central region protruded or receded relative to the surrounding annulus. Task difficulty varied depending on the disparity difference between the center and the surrounding annulus (Fig. 1A).

\section{Behavioral and V2 neuronal performance for the fine disparity discrimination task}

The animals learned to perform the fine disparity discrimination task with high accuracy: the mean psychophysical threshold (defined as $84 \%$ correct; see Material and Methods) was $0.032 \pm$ $0.042^{\circ}\left(0.044 \pm 0.045^{\circ}\right.$ and $0.022 \pm 0.036^{\circ}$ for Monkey 1 and Monkey 2, respectively). These values were comparable with those previously reported in macaques for the same task (Prince et al., 2000; Shiozaki et al., 2012). The behavioral data for one example session in each monkey is shown in Figure 2 (top row, black symbols; note that the disparity value of the surround is marked by the dashed vertical line). The activity of the simultaneously recorded single units changed substantially around the range of disparity values used during the behavioral task. For both units, the firing rate varied as monotonic function of disparity over the range used. We then used the mean responses on each trial to compute neurometric functions (see Materials and Methods), which we superimpose on the psychophysical functions (top panel, gray squares), and use to compute neurometric thresholds. The neurometric thresholds for Monkey 2 (Fig. 2, top right) slightly exceeded the simultaneously recorded psychophysical thresholds. This was typical in the population as can be seen in Figure 3. The mean \pm SD neurometric threshold across all units was $\left(0.051 \pm 0.070^{\circ}\right.$; it was $0.061 \pm 0.085^{\circ}$ and $0.043 \pm$ $0.055^{\circ}$ for Monkey 1 and Monkey 2, respectively). The mean ratio comparing the neurometric and psychophysical thresholds was ( $1.55 \pm 0.83$; for Monkey 1 and 2, it was $1.22 \pm 0.86$ and $2.20 \pm$ 0.66 , respectively). Across all sessions, we found a significant correlation between the psychophysical and neurometric thresholds (Spearman's rank $r=0.51, p<10^{-3}$ ). Several experimental
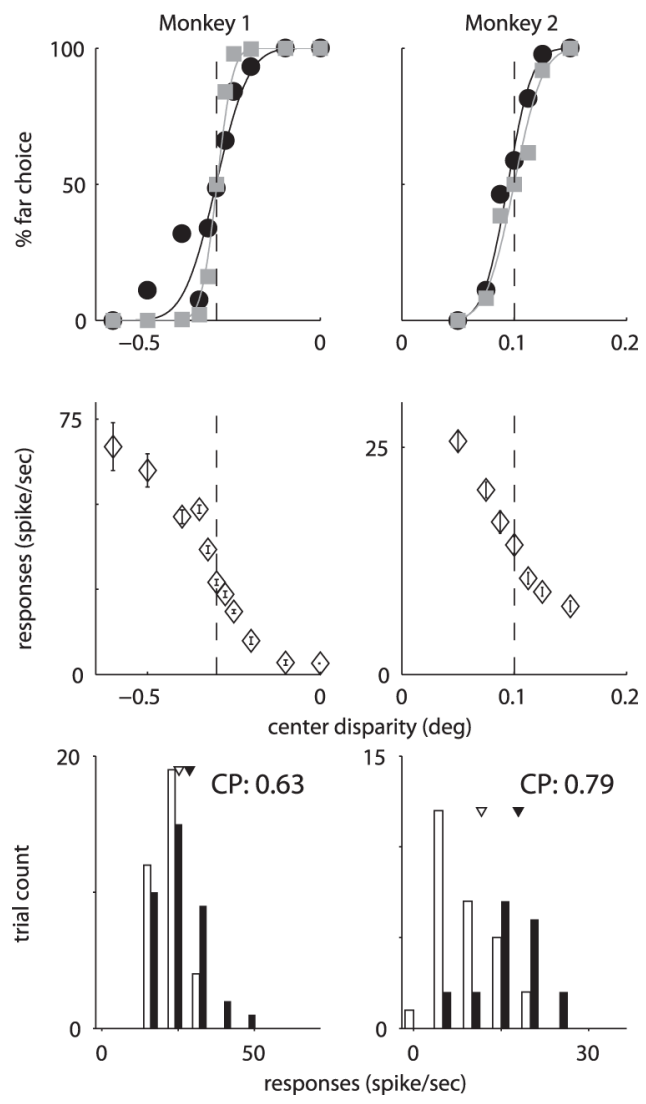

Figure 2. Example neuronal and behavioral responses from the 2 animals. Left column, Monkey 1. Right column, Monkey 2. Top, Black circles represent psychophysical performance as percentage far choices as a function of the disparity $\left(^{\circ}\right)$ of the stimulus center. Vertical dotted line indicates the disparity at which the surround was shown. Gray squares represent the neurometric curves of the simultaneously recorded neurons. Continuous lines indicate the cumulative Gaussian fits. Middle, The mean firing rate of the recorded neurons is plotted as a function of the disparity of the center. Both neurons change their firing rate monotonically around the disparity of the surround (vertical dashed line). Bottom, Distribution of firing rates (spikes/s) for $0 \%$ signal trials are shown separated by the animal's choice. Filled and open symbols represent choice toward a neuron's preferred and null disparity, respectively. Triangles represent the mean firing rates for each choice. The CPs for each neuron were 0.63 (left) and 0.79 (right).

covariates drove this correlation, of which the most important was the magnitude of the reference disparity (the background disparity), similar to Prince et al. (2000). The correlation between |background disparity| and psychophysical threshold (Spearman's rank $r=0.58, p<10^{-6}$, Monkey 1, $r=0.77, p<10^{-6}$, Monkey 2, $r=0.37, p=0.024$, and that between |background disparity| and neurometric threshold (Spearman's rank $r=0.54$, $p<10^{-5}$, Monkey 1, $r=0.65, p<10^{-4}$, Monkey 2, $r=0.39, p=$ $0.016)$ were of comparable magnitude.

Together, these analyses established that both monkeys performed the fine disparity discrimination with high precision and the recorded population of neurons in V2 provided signals suitable for this task.

\section{Decision-related activity in disparity-selective V2 neurons during the fine disparity discrimination task}

We therefore asked next whether the activity of the recorded neurons correlated with the monkeys' behavioral judgment on a trial-by-trial basis. For this, we divided the responses of each unit to the $0 \%$ signal stimulus (i.e., the disparity value of the center that was identical to that of the surround) into two groups: those for which the monkey chose the neuron's preferred and null dis- 

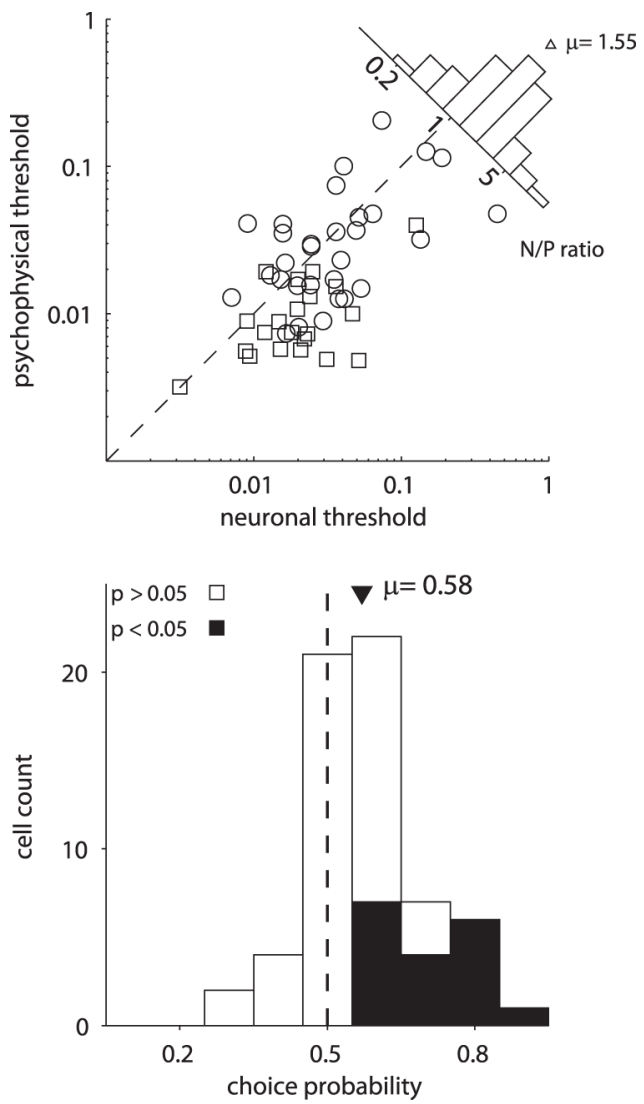

Figure 3. Population neuronal and behavioral data for the fine disparity discrimination task. Top, Neurometric thresholds for individual units are compared with the simultaneously measured psychometric thresholds. Circles represent Monkey 1 . Squares represent Monkey 2. $n=51$. The N/P ratios are displayed as a frequency histogram along the diagonal axis. The mean value is 1.55 , indicating that, on average, the neurometric thresholds are slightly worse than the performance of the animal. Bottom, The distribution of CPs for all $n=63$ units. Triangle represents the mean value (0.58), indicating that the mean is higher than chance level $(0.5$, dashed line). Filled bars represent cells $(n=18)$ with individually significant CPs.

parity. The distributions for two example units are shown in Figure 2 (bottom row). For both units, the distribution for choices toward the neuron's preferred disparity is slightly shifted toward higher values (Fig. 2, bottom row, filled bars). We then computed ROC curves based on these distributions and the area under the ROC curve was defined as CP (Britten et al., 1996). It corresponds to the probability with which an ideal observer would correctly predict the monkeys' choice at the end of the trial given two stimulus presentations in an interval forced choice, and the spike counts from each interval from the recorded neuron on each trial. For the two example neurons, CP was 0.62 and 0.79 , respectively. For each of these neurons, there was a significant trial-by-trial correlation between the activity of the individual unit and the monkey's behavioral choice $(p<0.05)$. On average, we also observed significant CPs across the population of recorded units. The distribution of CPs for all 63 single units is shown in Figure 3 (bottom). We found a mean CP significantly $>0.5$ (mean $=0.58, p<10^{-6}, t$ test). The mean CP was also significant in each monkey individually (Monkey 1 : mean $=0.58$ $p<0.001, n=37$; Monkey 2, mean $=0.58, p=0.0022, n=26)$. For a substantial fraction of units (18 of 63, 29\%), CP was individually significant. All of these units had CPs $>0.5$. These results indicate that there are systematic trial-by-trial correlations between neural activity of individual neurons and the animals'
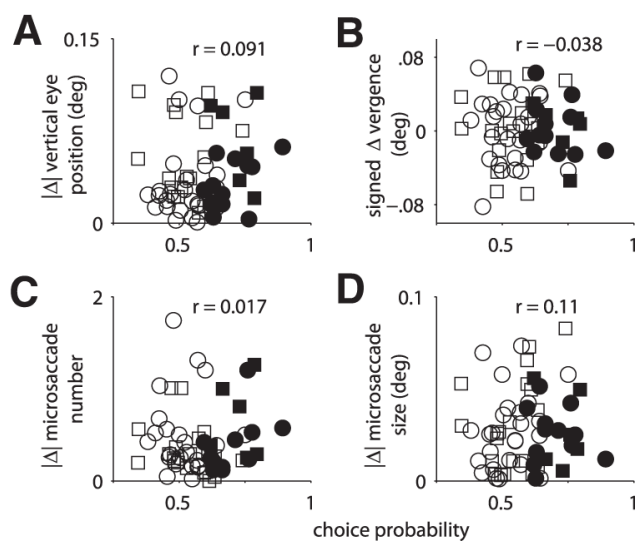

Figure 4. CPs cannot be explained by systematic differences in eye movements. Circles represent Monkey 1. Squares represent Monkey 2. Filled symbols represent units that had individually significant CPs $(p<0.05)$. $\boldsymbol{A}$, The absolute difference in mean vertical eye position as a function of choice is not significantly correlated with $C P(r=0.091, p=0.48)$. $\boldsymbol{B}$, We compare the difference in vergence, signed according to the neuron's preference, against $C P$ and find no significant correlation $(r=-0.038, p=0.76)$. $C$, The absolute difference in the mean number of microssacades between near and far choices is not significantly correlated with $C P(r=0.017, p=0.90)$. D , The absolute difference in average microsaccade size between near and far choices is plotted against $C P(r=0.11, p=0.11)$.

choices. The mean CP values are as large as those previously found for a fine disparity discrimination tasks in downstream areas V4 (mean CP 0.55) (Shiozaki et al., 2012) and IT (mean CP 0.56) (Uka et al., 2005).

\section{Control analyses to account for potential effects of eye movements}

Although the animals were required to maintain precise fixation during the stimulus presentation and although we sought to minimize systematic differences in eye position with choice (see Materials and Methods), small systematic differences in eye movements with choice could in principle give rise to significant trial-by-trial correlations between the activity of single units and the animals' choices. We therefore performed a number of control analyses to verify that such effects could not account for the CPs we observed.

\section{Mean eye position}

Because the animals were required to indicate their choices with a vertical saccade toward a target above or below the fixation point, anticipatory eye movements within the fixation window in the direction of the choice target could potentially result in systematic mean differences in eye position with choice. To verify that our attempt to minimize systematic differences with choice (see Materials and Methods) was successful, we computed the average vertical eye position during the stimulus presentation. For each unit, we then compared the average absolute vertical eye position differences between near and far choices. The remaining mean absolute eye position difference was small, $0.041 \pm 0.033^{\circ}$ (Fig. $4 A$ ). Furthermore, there was no significant correlation between $\mathrm{CP}$ and absolute vertical eye position differences $(n=63$ units, $r=0.091, p=0.48)$. These results were similar in each monkey individually (Monkey $1: n=37$ units, mean $=0.034 \pm 0.029, r=$ $0.17, p=0.32$; Monkey 2: $n=26$ units, mean $=0.052 \pm 0.034$, $r=0.0092, p=0.96)$. Together, these results suggest that systematic differences in vertical eye position as function of choice cannot explain the CPs we observed. Similarly, choice-related differences in horizontal eye position were not correlated with CP 
(both animals: mean $=0.034 \pm 0.028, n=63$ units, $r=-0.023$, $p=0.86$; Monkey 1: $n=37$ units, mean $=0.032 \pm 0.022, r=$ $0.084, p=0.62$; Monkey 2: $n=26$ units, mean $=0.037 \pm 0.035$, $r=-0.12, p=0.57)$, data not shown).

\section{Vergence}

Changes in an animal's vergence will cause a corresponding change in the absolute disparity of the stimulus. We found that the mean difference in vergence across all sessions was small $\left(\right.$ mean $=0.0063 \pm 0.034^{\circ} ;$ Monkey $1:$ mean $=0.0086 \pm 0.032^{\circ}$, SD; Monkey 2: mean $\left.=0.0030 \pm 0.036^{\circ}\right)$, but it indicated that the animals converged slightly more on near choices compared with far choices. Although these choice-dependent differences are small, they could result in choice-dependent changes in firing rate. A choice-dependent difference in vergence would yield opposite effects on CP for near and far-preferring neurons. For far-preferring neurons, stronger divergence on far choices than on near choices would result in a larger far disparity on far choices and hence in a $\mathrm{CP}>0.5$. In contrast, the same choice-dependent difference in vergence would cause a $\mathrm{CP}$ of $<0.5$ for a nearpreferring neuron. To take this differential effect of vergence with respect to a neuron's tuning preference into account, we examined whether CP depended on the vergence difference between the animal's choices to the preferred and null disparity for each neuron (Fig. 4B). Specifically, we changed the sign of the vergence difference ( $\Delta$ vergence) according to the preference of the recorded neuron: a positive $\Delta$ vergence corresponds to stronger divergence on near choices for near-preferring neurons, and to a stronger divergence on far choices, for far-preferring neurons. Using this signed vergence difference, positive values would be expected to produce CPs $>0.5$. We found that far-preferring neurons showed a positive trend between the mean difference in vergence and CP $(r=0.41, p=0.066, n=21)$. However, for near-preferring neurons, the trend was in the opposite direction $(r=-0.16, p=0.33, n=42)$. Indeed, across all neurons, the correlation between the mean signed vergence difference and CP was $-0.038(p=0.76, n=63 ; r=-0.052, p=0.76, n=37$ in Monkey 1 and $r=-0.020, p=0.92, n=26$ in Monkey 2). Thus, overall, the weak correlation between CP and choice-related vergence changes is in the wrong direction to account for the observed CP.

\section{Microsaccades}

Microsaccades transiently modulate neuronal responses (Gur et al., 1997; Bair and O'Keefe, 1998; Leopold and Logothetis, 1998; Martinez-Conde et al., 2000; Hass and Horwitz, 2011; McFarland et al., 2015). Systematic differences in the frequency, size, or direction of microsaccades with choice might thus result in systematic correlations between a neuron's firing rate and choice. To verify that such an effect could not explain the CPs we observed, we correlated each of these metrics with CP. We found no systematic relationship between the /difference in the number of microsaccades $\mid$ as a function of choice and $\mathrm{CP}$ across animals or in either animal alone (Fig. $4 C ; r=0.017, p=0.90, n=63$; Monkey 1: $r=-0.057, p=0.74, n=37$; Monkey 2: $r=0.14, p=$ $0.50, n=26)$. Similarly, there was no significant correlation between CPs and the choice-dependent $/$ difference in size of microsaccades (Fig. 4D; $r=0.11, p=0.37, n=63$; Monkey 1: $r=$ 0.069, $p=0.69, n=37$; Monkey 2: $r=0.17, p=0.42, n=26$ ). Finally, the difference in the direction of saccades between far and near choices was not significantly correlated with CP $(r=-0.11$, $p=0.40, n=63$; Monkey 1: $r=-0.13, p=0.44, n=37$; Monkey 2: $r=-0.083, p=0.69, n=26$; data not shown).
Together, these additional analyses make it very unlikely that one of these different types of eye movements can explain the systematic differences in firing rate with choice we observed.

\section{Does decision-related activity depend on the V2 neurons' selectivity for relative disparity?}

Psychophysical studies in humans (Westheimer, 1979; McKee and Levi, 1987) and macaques (Prince et al., 2000) found that discrimination thresholds for small changes in disparity increase $\sim 10$-fold in the absence of a reference stimulus. This supports the view that fine disparity discrimination (as used in this study) relies on relative disparity judgments (i.e., the comparison of the disparity in nearby stimuli; e.g., the center vs the surrounding annulus in the stimulus we used). While the tuning of disparityselective neurons in V1 exclusively depends on the disparity presented inside the receptive field (absolute disparity tuning), a subset of disparity-selective neurons in V2 are selective for relative disparities. That is, their disparity selectivity changes systematically as a function of the disparity of a surrounding reference stimulus. To quantify the degree to which the disparity-selective neurons were selective for relative or absolute disparities, we measured the disparity tuning curve to the center of the stimulus for a set of different disparity values of the surround (Fig. 5, insets). The resulting response surfaces were then fit with a $2 \mathrm{D}$ Gabor function. We used the orientation of the Gabor to quantify the degree to which a neuron was selective for absolute or relative disparity. If the disparity tuning to the center was independent of the surround (i.e., the neuron showed absolute disparity tuning), the 2D Gabor was oriented vertically (90 degrees; Fig. 5, top two insets, two examples). Conversely, if the orientation of the Gabor was at 45 degrees, it means that a neuron was selective to a step in disparity relative to the surround that was, over a range of surround disparities, independent of the disparity of the surround. Examples for such relative disparity tuning are shown in Figure 5 (right, insets).

We previously proposed that cortical sensory neurons that show decision-related activity are organized in clusters or columns for the task-relevant feature (Nienborg and Cumming, 2014). We therefore wondered whether there was evidence for clustering for relative disparity in V2. To address this question, we compared the orientation of the $2 \mathrm{D}$ Gabor fit to the $2 \mathrm{D}$ response surface for changing center and surround disparities recorded for the single unit activity and the simultaneously recorded multiunit activity excluding the single-unit (Fig. 6). (The mean firing rate for the multiunit activity for the disparity tuning curves was $46 \mathrm{sp} / \mathrm{s}$, compared with a mean firing rate of $30 \mathrm{sp} / \mathrm{s}$ for the disparity tuning curves of the single unit activity, suggesting that, on average, the multiunit activity could be the sum of as few as two neurons.) The correlation was 0.66 (circular correlation, $p=0.017, n=35$ ), in support of a clustered organization for relative disparity tuning, although this correlation only reached significance in one of the animals (Monkey 1: circular correlation $=0.20, p=0.56, n=21$; Monkey 2: $0.80, p=0.014$, $n=14)$.

To address the question of whether decision-related activity depended on a neuron's tuning to relative disparity, we plotted the angle of the 2D Gabor against CP. First, although there was a subset of neurons that showed clear selectivity for relative disparity, the majority of neurons were tuned to absolute disparities, consistent with previous findings (Thomas et al., 2002). Second, given the psychophysical evidence that subjects rely on relative disparity judgments for this task, one might expect that neurons selective for relative disparity have higher CPs. If this were the 


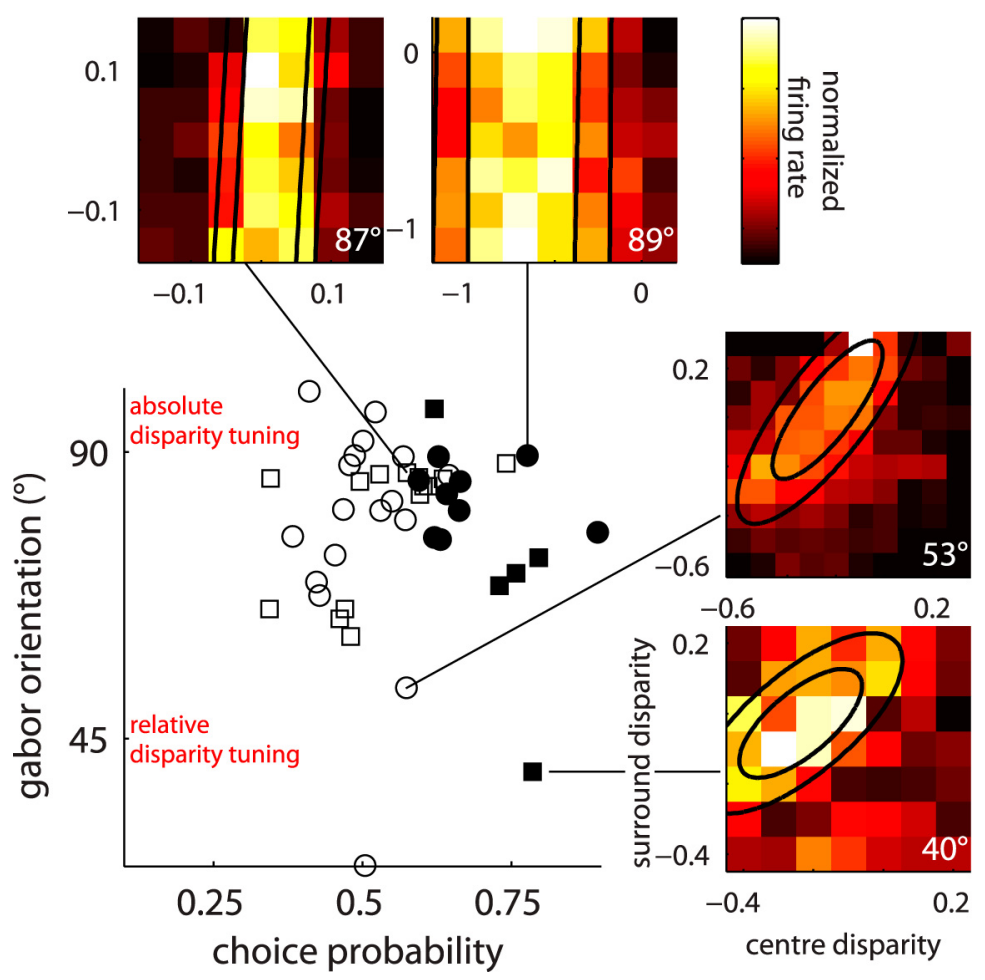

Figure 5. CP does not depend on a neuron's degree of selectivity for relative disparity. For each neuron, disparity selectivity to the stimulus center (abscissa of insets) was measured for several disparities of the surrounding annulus (Insets, ordinate). The resulting $2 \mathrm{D}$ map was fitted with a $2 \mathrm{D}$ Gabor function. The orientation of the $2 \mathrm{D}$ Gabor was then used to estimate a neuron's selectivity for relative disparity. Top two insets, For neurons selective to absolute disparity, the orientation was close to $90^{\circ}$. Right two insets, For neurons selective for relative disparity, the orientation was tilted toward $45^{\circ}$. There is no systematic relationship between the orientation of the 2D Gabor ( $y$-axis) and CP (Spearman's rank $r=0.076, p=0.62, n=45$ ). Circles represent Monkey 1. Squares represent Monkey 2. For filled data points, the CP was significant $(p<0.05)$.

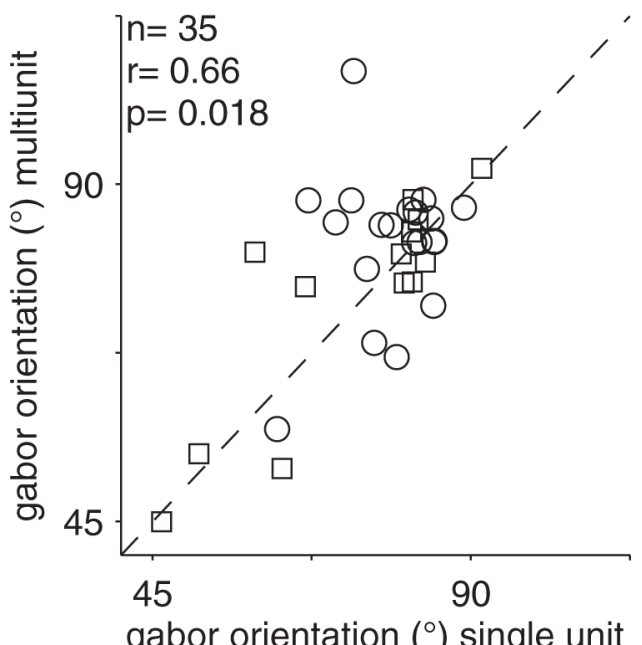

Figure 6. Similarity of the degree of tuning for relative disparity for single-unit and multiunit activity. The orientation of the 2D Gabor fit to the 2D disparity tuning map (compare Fig. 5) for the single unit (abscissa) is plotted against that obtained for the simultaneously recorded multiunit activity (ordinate). Note the correlation between these values $(r=0.66, p=0.018$, circular correlation).

case, we should observe a negative correlation between the two metrics. However, our data do not support this hypothesis. There was no significant correlation between these two parameters (Spearman's rank $r=0.077, p=0.62, n=45$ ). This is also the case when tested in each animal individually (Monkey 1: Spear- man's rank $r=0.10, p=0.63, n=26$; Monkey 2: Spearman's rank $r=0.088$, $p=0.72, n=19)$. Indeed, for the subset of neurons for which the orientation of the 2D Gabor was significantly $<90$ degrees, in support of significant relative disparity tuning $(n=31)$, the $\mathrm{CP}($ mean $\mathrm{CP}=0.58)$ was not significantly different from neurons in which orientation of the 2D Gabor was statistically indistinguishable from or exceeded $90^{\circ}(n=14$, mean $\mathrm{CP}=0.56$, $p=0.934, t$ test). This indicates that decision-related activity is independent of a neuron's selectivity for relative disparity.

Similarly, the neurometric thresholds were not significantly different between groups $(p=0.65$, Wilcoxon rank sum test; the mean \pm SD neurometric threshold was $0.036 \pm 0.031$ degrees for the group of neurons whose 2D Gabor orientation was significantly $<90$ degrees and $0.090 \pm 0.13$ degrees for the remaining neurons).

\section{Comparing CPs for the fine and coarse disparity discrimination task}

Our animals also had received extensive training on a coarse disparity discrimination task (Fig. 1B), and we have previously reported CPs for disparity-selective neurons in V2 for this coarse discrimination task. Given the surprising independence of $\mathrm{CP}$ in the fine disparity discrimination task and a neuron's selectivity for relative disparity, we wondered whether there was any relationship between the CPs across the two tasks (Fig. 1A,B). For example, if the animals' perceptual strategy were largely constant across tasks and dominated by the coarse disparity discrimination task, this might lead to a correlation of CPs between tasks and obscure a relationship between a neurons' tuning for relative disparity and $\mathrm{CP}$ for the fine task. We therefore additionally recorded the neural activity while the animals performed the coarse disparity discrimination task. For a subset of cells ( $n=25$; Monkey 1: $n=15$; Monkey 2: $n=10$ ), our neuronal and behavioral inclusion criteria were met for both tasks. For these cells, we compare the CPs for the fine task (abscissa) with those for the coarse task (ordinate) in Figure 7. There was a weak but not statistically significant correlation of the CPs for the two tasks $(r=0.38, p=0.064, n=$ 25; Monkey 1: $r=0.52, p=0.046, n=15$; Monkey 2: $r=0.26$, $p=0.47, n=10)$. These estimates of CP were performed on a median number of trials for the $0 \%$ signal condition of $n=46$ for the fine and $n=83$ for the coarse disparity discrimination task, respectively. To improve our statistical power in estimating $\mathrm{CP}$, we also computed the correlation between the "grand" CPs (compare Britten et al., 1996) for both tasks. These also revealed a weak correlation $(r=0.30, p=0.15, n=25)$ that did not reach statistical significance. This suggests that the absence of a relationship between the neuronal tuning for relative disparity and $\mathrm{CP}$ for the fine disparity discrimination task does not result from the animals' perceptual strategy being dominated by the coarse disparity discrimination task. As the sample size is small, we cannot exclude a modest correlation between CPs for the two tasks, 


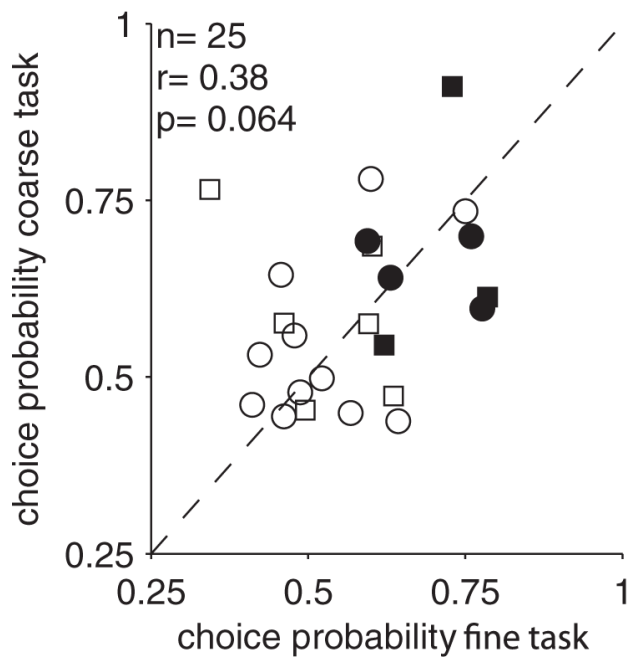

Figure 7. Comparison of CPs for the fine and coarse disparity discrimination task. For a subset of neurons $(n=25)$, we recorded from the same unit while the animals performed the two tasks consecutively. Filled symbols, CP was significant $(p<0.05)$ in the fine disparity discrimination task. The CP obtained for the fine disparity discrimination task (abscissa) is compared with that for which the monkey performed the coarse discrimination task (ordinate). The correlation between the (Ps for the two tasks is not significant $(r=0.38, p=0.064)$.

but the weak correlation does not seem compatible with the same readout being used for both tasks.

\section{Testing theoretical predictions of optimal linear readout in the presence of information-limiting correlations for both tasks}

Although previous work (Shadlen et al., 1996; Cohen and Newsome, 2009; Haefner et al., 2013) has highlighted the fact that interneuronal correlations ("noise correlations"; for review, see Cohen and Kohn, 2011) complicate the interpretation of CPs, recent theoretical studies (Haefner et al., 2013; Moreno-Bote et al., 2014; Pitkow et al., 2015) have refined our understanding of the relationship. In particular, Moreno-Bote et al. (2014) showed that noise correlations only of a particular type limit the information that can be read out from a population of sensory neurons. These correspond to interneuronal correlations proportional to the product of the derivative of the neurons' tuning curves and are termed "information-limiting" correlations (Moreno-Bote et al., 2014). Using these insights, Pitkow et al. (2015) showed that, if there are information-limiting correlations in a population of neurons, and decisions are based on an optimal linear decoder of that population, then a unique relationship holds between $\mathrm{CP}$ and the ratio (psychometric threshold)/(neurometric threshold). They found that, for a heading discrimination task, the neuronal data from early sensory areas were consistent with this relationship (Pitkow et al., 2015). If optimal linear readout of activity in early sensory areas provides a good account of psychophysical performance, the data we present here should follow this relationship. The results reported here, combined with our previous results in V2 for the coarse disparity discrimination task performed by the same animals (Nienborg and Cumming, 2006, 2007), allow us to test this prediction in an early visual area across two different but closely related tasks.

First, we explored whether the predictions by Pitkow et al. (2015) for an optimal linear decoder and information-limiting correlations held for the coarse disparity discrimination task. Although we found a significant positive correlation between our measured choice correlations and those predicted supporting a

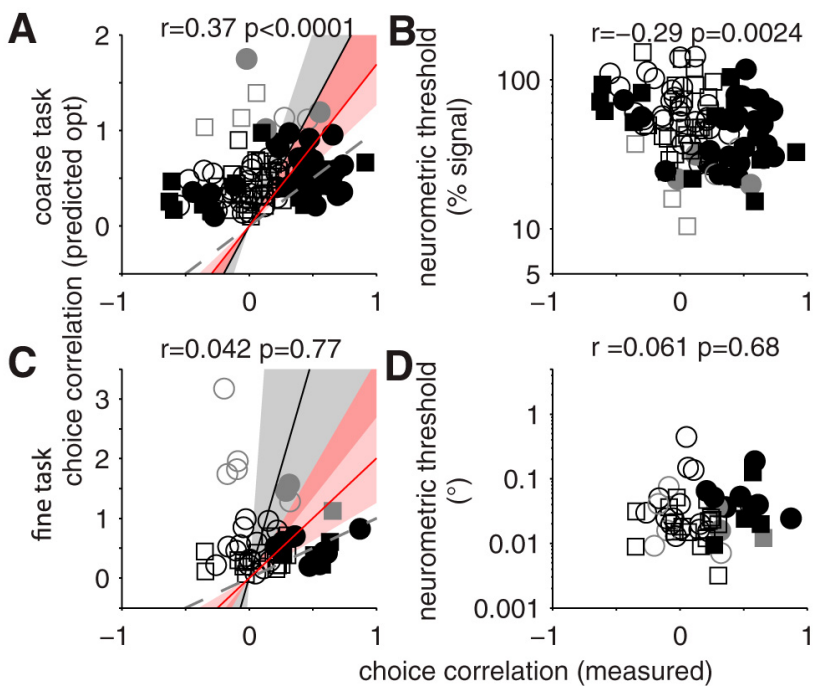

Figure 8. Testing predictions for an optimal linear readout of a neural population with information-limiting noise across two tasks in V2. $\boldsymbol{A}, \boldsymbol{B}$, Data for the coarse disparity discrimination task are shown. A subset of this dataset was previously included in Nienborg and Cumming $(2006,2007)$. $\boldsymbol{A}$, The measured choice correlation is correlated with that predicted for an optimal linear readout and information-limiting correlations based on the psychophysical and neurometric thresholds (Spearman's rank $r=0.37, p<10^{-4}, n=104$ ). The best fitting slope (black solid) is 2.50 ( $\mathrm{Cl}:[1.73,4.10]$, gray shading), deviating significantly from unity (dashed line). When excluding data points for which the monkeys' performance was suboptimal (the predicted choice correlation exceeds 1 , gray symbols), the best fitting slope (red solid) is 1.70 (Cl: $[1.27,2.53]$, red shading). $\boldsymbol{B}$, Choice correlation is negatively correlated with the neurometric thresholds (Spearman's rank $r=-0.29, p=0.0024, n=104$ ). $\boldsymbol{C}, \boldsymbol{D}$, The analogous data as in $\boldsymbol{A}, \boldsymbol{B}$ are shown for the fine disparity discrimination task. For both comparisons, there is no significant correlation with choice correlation (C: Spearman's rank $r=0.04, p=0.77, n=51$; $D$ : Spearman's rank $r=0.061, p=0.68, n=51)$. The best fitting slope (black solid) is 7.36 ( $\mathrm{Cl}$ : $[2.69,28.94]$, gray shading), as well as that when excluding data for which the monkeys' performance was suboptimal (the predicted choice correlation exceeds 1, gray symbols; best fitting slope is $2.01, \mathrm{Cl}:[1.28,3.86]$, red solid and red shading) deviate significantly from unity (dashed line).

qualitative agreement with the predictions (see Materials and Methods; Spearman's rank $r=0.37, p<10^{-4}, n=104$; Monkey 1: Spearman's rank $r=0.38, p=0.0021, n=62$; Monkey 2: Spearman's rank $r=0.31, p=0.047, n=42$; Fig. $8 A$ ), similar to Pitkow et al. (2015, their Fig. 6C, top), the observed values are systematically lower than predicted. Sessions for which the psychophysical threshold significantly exceeds the neurometric threshold imply suboptimal readout by themselves and may reflect poor behavioral performance due to reasons, such as lack of motivation. To account for this, we also restricted our analysis to sessions for which the psychophysical performance is better than the neuronal sensitivity by excluding sessions for which $\mathrm{P} / \mathrm{N}>1$ (Fig. 8A, gray data points). The resulting best fitting curve (red line) still significantly deviates from unity (red represents CIs), indicating that the predicted choice correlations for optimal linear readout are systematically higher than those measured. We also observed a negative correlation between choice correlations and the neurometric threshold (Spearman's rank $r=-0.29, p=$ 0.0024, $n=104$; Monkey 1: Spearman's rank $r=-0.38, p=$ 0.0027, $n=62$; Monkey 2: Spearman's rank $r=-0.14, p=0.36$, $n=42$; Fig. $8 B$ ), as often found for perceptual discrimination tasks (Celebrini and Newsome, 1994; Britten et al., 1996; Romo et al., 2002; Uka and DeAngelis, 2004; Purushothaman and Bradley, 2005; Gu et al., 2008; Liu et al., 2013a, b; Nienborg and Cumming, 2014). Contrasting with the results for the coarse disparity discrimination task, we found that there was neither a significant 
correlation between predicted and observed choice correlations (Spearman's rank $r=0.042, p=0.77, n=51$; Monkey 1: Spearman's rank $r=-0.20, p=0.29, n=30$; Monkey 2: Spearman's rank $r=0.41, p=0.065, n=21$; Fig. $8 C$ ), nor a significant correlation between choice correlation and the neurometric threshold (Spearman's rank $r=0.061, p=0.67, n=51$; Monkey 1: Spearman's rank $r=0.14, p=0.45, n=30$; Monkey 2: Spearman's rank $r=-0.022, p=0.93, n=21$; Fig. $8 D$ ). (We verified that the absence of a significant correlation in Fig. $8 D$ could not be explained by the influence of the background disparity on the neurometric threshold. The partial correlation between neurometric threshold and choice correlation remained nonsignificant when accounting for the effect of |background disparity| (partial rank correlation, $r=0.078, p=0.590$.) Similarly to the results for the coarse disparity discrimination task, the observed choice correlations were lower than those predicted for the fine disparity discrimination task (Fig. 8C, black solid line), even when restricting the analysis to sessions for which the psychophysical performance exceeded the neuronal sensitivity (Fig. $8 C$, red solid line).

We next wondered whether the relationship predicted for optimal linear readout in the presence of information-limiting correlations may apply early in the trial, during which choice correlations are thought to be dominated by bottom-up processing compared with late in the trial, when they are thought to also reflect feedback signals (Wimmer et al., 2015). We therefore repeated the analyses for the fine and coarse disparity discrimination task using the neural data for only the first $500 \mathrm{~ms}$ or last 500 $\mathrm{ms}$ in each trial. Because we only had measurements of the psychophysical threshold for $2 \mathrm{~s}$ stimulus presentations, we adjusted these, assuming a weighted integration of the stimulus information as previously reported for the coarse disparity discrimination task (Nienborg and Cumming, 2009). These additional analyses revealed that for neither task or time interval did the choice correlations match those predicted for optimal linear readout of a population with information-limiting correlations (Fig. 9). The results were similar when assuming perfect integration (data not shown).

Thus, although choice correlation for the coarse task followed the pattern predicted for optimal linear readout in the presence of information-limiting correlations, for neither task did the predicted relationship hold quantitatively.

\section{Discussion}

The goal of this study was to determine whether disparityselective neurons in visual area V2 show decision-related activity (CPs) for a fine disparity discrimination task, to understand the origin of CP for this task in downstream areas. Psychophysical results suggest that this fine disparity discrimination relies on relative disparity judgments, and V2 is the first area in the visual processing hierarchy known to have a population of neurons selective for relative disparity (Cumming and Parker, 1999; Thomas et al., 2002). We found that neurons in V2 were very well suited for the task. Indeed, although the discrimination performance of individual neurons was on average somewhat worse than that of the animal, the most selective neurons exceeded the discrimination performance of the animals. Additionally, the observed neuronal discrimination performance substantially exceeded that previously reported for visual area V4 in the same task, by approximately an order of magnitude (Shiozaki et al., 2012). Moreover, we observed decision-related activity for this task in V2, with a mean CP of 0.58 , which weakly exceeded that previously observed for visual area V4 (0.55). Our results therefore make $\mathrm{V} 2$ the earliest stage in the visual hierarchy for which

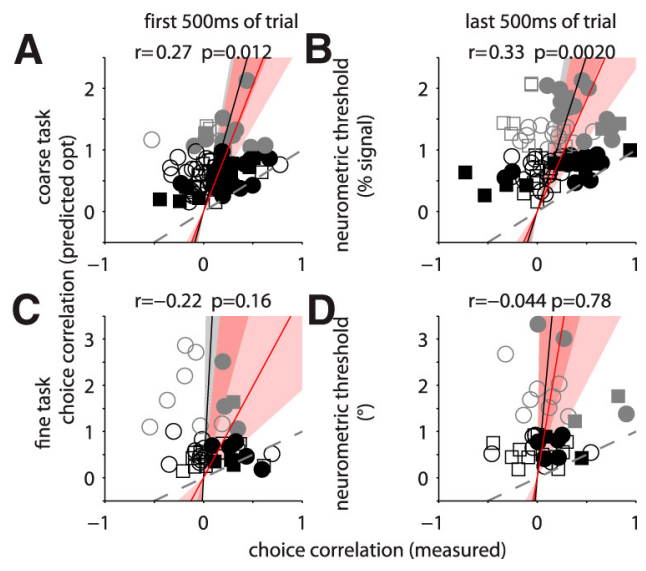

Figure 9. The predictions for optimal linear readout of the V2 population with informationlimiting noise also fail for the first and last $500 \mathrm{~ms}$ of each trial. For each panel, neural data for the respective $500 \mathrm{~ms}$ bin during the trial are considered. The corresponding psychometric thresholds are scaled assuming integration during the respective $500 \mathrm{~ms}$ bins of the visual information according to previously measured weights (Nienborg and Cumming, 2009). The results were similar to those assuming perfect integration (data not shown). $\boldsymbol{A}, \boldsymbol{B}$, The same analysis as in Figure $8 A$ is shown but for the data in the first $(\boldsymbol{A})$ or last $(\boldsymbol{B}) 500 \mathrm{~ms}$ bin of each trial. The best fitting slopes ( $\boldsymbol{A}$ : black solid: 5.61, Cl: [3.89, 8.97], red solid: $4.11 \mathrm{Cl}$ : [2.78, 8.04] B: black solid: $5.61 \mathrm{Cl}$ : $[3.75,7.36]$, red solid: $3.63 \mathrm{Cl}$ : $[2.32,9.13])$ deviate significantly from unity. $\boldsymbol{C}, \boldsymbol{D}$, The same analysis as in Figure $8 B$ is shown but for the data of the first $(\boldsymbol{C})$ or last $(\boldsymbol{D})$ $500 \mathrm{~ms}$ bin of each trial. As for the coarse disparity discrimination task, the best fitting slopes ( $\boldsymbol{A}$ black: $39.31 \mathrm{Cl}$ : $\left[7.68,10^{8}\right]$, red: $3.96 \mathrm{Cl}$ : [1.91, 21.33]; $\boldsymbol{D}$ : black: $23.18 \mathrm{Cl}:\left[8.05,10^{6}\right]$, red: 12.77 $\left.\mathrm{Cl}:\left[4.12,10^{7}\right]\right)$ deviate significantly from the predictions for optimal linear readout in the presence of information-limiting correlations.

decision-related activity has been reported for this task. Whether V4 inherits this activity from V2 could depend upon whether disparity-selective cells in V2 project to V4. Disparity selectivity is found mostly within the thick stripes of V2, which are not the main source of projections from V2 to V4. We cannot be certain whether the neurons we recorded project to $\mathrm{V} 4$ or whether they were in the thick stripes.

Interestingly, the degree to which V2 neurons showed decision-related activity did not correlate with their tuning for relative disparity. At first sight, the absence of a relationship between CP and a neuron's selectivity for relative disparity is surprising. But extensive computational (Shadlen et al., 1996; Cohen and Newsome, 2009), analytical (Haefner et al., 2013; Pitkow et al., 2015), and experimental (Liu et al., 2013b) work has highlighted that CPs depend on correlations between neurons in the population. Because these correlations cannot be explained by changes in the stimulus, they are often referred to as "noise correlations" (for review, see Cohen and Kohn, 2011). Thus, provided neurons with and without selectivity for relative disparity show appropriate noise correlations, these results may be reconciled with simple pooling models. It is also important to note that neurons selective only for absolute disparity may nonetheless provide the critical input to a later computation of relative disparity. Indeed, the neurometric thresholds of neurons in V1 for absolute disparity are comparable with psychometric judgments of relative disparity (Prince et al., 2000). It may be for this reason that we find no difference in neurometric threshold between neurons selective for absolute or relative disparity in V2.

Additionally, we found that, for both tasks, the responses of V2 neurons are not quantitatively compatible with the theoretical predictions developed by Pitkow et al. (2015) for an optimal linear readout of a neuronal population containing information-limiting correlations, although the results for the coarse task followed the 
predicted pattern qualitatively. Because the neurometric and psychophysical threshold were similarly affected by day-to-day changes in the stimulus, such as the background disparity (compare Fig. 3, top), the failure to predict choice correlations for the fine disparity discrimination task is unlikely due to stimulus-induced variability of neuronal thresholds. This poses important questions about the origin of CP in downstream areas. One possibility is that a nonlinear transformation of the signals from V2 is used to construct downstream responses, and then a linear decoder is applied to those downstream areas. Under this hypothesis, the current theoretical framework, based on pooling, makes no quantitative predictions about CP in V2. This poses a significant challenge, best exemplified by neurons that are not sensitive to relative disparity, and yet show $\mathrm{CP}$ in the fine task (Fig. 5). A nonlinear transformation of these signals is required to represent relative disparity, thought to be used for making these fine judgments. If simple pooling of these responses does not explain behavior, then we have no quantitative account of $\mathrm{CP}$ in these neurons. Previously, it might have been possible to think that a linear decoding of a downstream population is approximated by a linear decoding of V2. The fact that CP does not follow the predicted relationship (Fig. 8) demonstrates that this approximation is not applicable. It is also important to note, that under the demands of the task we used, a relative disparity representation is not a requirement (because the background disparity was held constant), so that a linear readout of $\mathrm{V} 2$ neurons could, in principle, have been used to perform the task. This is similar to results reported for Otolith afferent fibers when animals report the direction of translation in the dark (Yu et al., 2015). The relationship between CP and psychophysical and neurometric performance does not follow the predictions of linear decoding, but there are reasons to believe that nonlinear operations are required to generate a useful sensory representation (Yu et al., 2015; Cumming and Nienborg, 2016).

A second possibility is that $\mathrm{CP}$ does not simply reflect the consequences of noise in afferent sensory neurons but arises in part because a component of noise correlations results from topdown processes, as empirical and analytical evidence increasingly suggests (Roelfsema et al., 2004; Cohen and Newsome, 2008; Cohen and Maunsell, 2009, 2011; Mitchell et al., 2009; Gu et al., 2011; Ruff and Cohen, 2014a, b; Rabinowitz et al., 2015). Indeed, the framework by Pitkow et al. (2015) relies on theoretical work by Haefner et al. (2013), which requires that the correlated sensory signals are read out without any information about the sources of the correlation. That is, this theoretical work does not allow for any centrally generated correlations in sensory neurons that are discounted when forming a perceptual decision (e.g., because they reflect postdecision signals). It is possible that such postdecision top-down signals late in the trial disrupt the predicted relationship between $\mathrm{CP}$ and the $\mathrm{P} / \mathrm{N}$ ratio, even if the sensory activity early in the trial (predecision) is read out by an optimal linear decoder in the presence of information-limiting correlations. The dichotomy "early predecision" versus "late postdecision" is a simplification used here for clarity. Moreover, our analysis in Figure 9 does not suggest that such a simple distinction applies. However, more plausible proposals for the dynamics of these signals exist (Wimmer et al., 2015; Haefner et al., 2016).

Together, our results are compatible with the view that sensory signals in V2 are used for fine disparity judgments in addition to coarse disparity judgments. But the data are not compatible with a simple linear decoding of responses in V2. This discrepancy may reflect variability in how the task-relevant top-down beliefs (Haefner et al., 2016) are aligned with the different tasks. Alternatively, it may reflect the importance of downstream computation in forming a perceptually relevant representation. In both cases, these results highlight the need for models more sophisticated than optimal linear decoders to account for decision-related activity in sensory neurons.

\section{References}

Bair W, O'Keefe LP (1998) The influence of fixational eye movements on the response of neurons in area MT of the macaque. Vis Neurosci 15:779786. Medline

Britten KH, Newsome WT, Shadlen MN, Celebrini S, Movshon JA (1996) A relationship between behavioral choice and the visual responses of neurons in macaque MT. Vis Neurosci 13:87-100. CrossRef Medline

Celebrini S, Newsome WT (1994) Neuronal and psychophysical sensitivity to motion signals in extrastriate area MST of the macaque monkey. J Neurosci 14:4109-4124. Medline

Chen G, Lu HD, Roe AW (2008) A map for horizontal disparity in monkey V2. Neuron 58:442-450. CrossRef Medline

Cohen MR, Kohn A (2011) Measuring and interpreting neuronal correlations. Nat Neurosci 14:811-819. CrossRef Medline

Cohen MR, Maunsell JH (2009) Attention improves performance primarily by reducing interneuronal correlations. Nat Neurosci 12:1594-1600. CrossRef Medline

Cohen MR, Maunsell JH (2011) Using neuronal populations to study the mechanisms underlying spatial and feature attention. Neuron 70:11921204. CrossRef Medline

Cohen MR, Newsome WT (2008) Context-dependent changes in functional circuitry in visual area MT. Neuron 60:162-173. CrossRef Medline

Cohen MR, Newsome WT (2009) Estimates of the contribution of single neurons to perception depend on timescale and noise correlation. J Neurosci 29:6635-6648. CrossRef Medline

Crapse TB, Basso MA (2015) Insights into decision making using choice probability. J Neurophysiol 114:3039-3049. CrossRef Medline

Cumming BG, Nienborg H (2016) Feedforward and feedback sources of choice probability in neural population responses. Curr Opin Neurobiol 37:126-132. CrossRef Medline

Cumming BG, Parker AJ (1999) Binocular neurons in V1 of awake monkeys are selective for absolute, not relative, disparity. J Neurosci 19:5602-5618. Medline

DeAngelis GC (2009) Roles of visual area MT in depth perception. In: The cognitive neurosciences Gazzaniga MS, ed), pp 483-498. Cambridge, MA: Massachusetts Institute of Technology.

Gu Y, Angelaki DE, Deangelis GC (2008) Neural correlates of multisensory cue integration in macaque MSTd. Nat Neurosci 11:1201-1210. CrossRef Medline

Gu Y, Liu S, Fetsch CR, Yang Y, Fok S, Sunkara A, DeAngelis GC, Angelaki DE (2011) Perceptual learning reduces interneuronal correlations in macaque visual cortex. Neuron 71:750-761. CrossRef Medline

Gur M, Beylin A, Snodderly DM (1997) Response variability of neurons in primary visual cortex (V1) of alert monkeys. J Neurosci 17:2914-2920. Medline

Haefner RM, Berkes P, Fiser J (2016) Perceptual decision-making as probabilistic inference by neural sampling. Neuron 90:649-660. CrossRef Medline

Haefner RM, Gerwinn S, Macke JH, Bethge M (2013) Inferring decoding strategies from choice probabilities in the presence of correlated variability. Nat Neurosci 16:235-242. CrossRef Medline

Hass CA, Horwitz GD (2011) Effects of microsaccades on contrast detection and V1 responses in macaques. J Vis 11:1-17. CrossRef Medline

Judge SJ, Richmond BJ, Chu FC (1980) Implantation of magnetic search coils for measurement of eye position: an improved method. Vision Res 20:535-538. CrossRef Medline

Krug K (2004) A common neuronal code for perceptual processes in visual cortex? Comparing choice and attentional correlates in V5/MT. Philos Trans R Soc Lond B Biol Sci 359:929-941. CrossRef Medline

Krug K, Parker AJ (2011) Neurons in dorsal visual area V5/MT signal relative disparity. J Neurosci 31:17892-17904. CrossRef Medline

Leopold DA, Logothetis NK (1998) Microsaccades differentially modulate neural activity in the striate and extrastriate visual cortex. Exp Brain Res 123:341-345. CrossRef Medline

Liu S, Dickman JD, Newlands SD, DeAngelis GC, Angelaki DE (2013a) Reduced choice-related activity and correlated noise accompany perceptual deficits following unilateral vestibular lesion. Proc Natl Acad Sci U S A 110:17999-18004. CrossRef Medline 
Liu S, Gu Y, DeAngelis GC, Angelaki DE (2013b) Choice-related activity and correlated noise in subcortical vestibular neurons. Nat Neurosci 16: 89-97. CrossRef Medline

Lu HD, Chen G, Tanigawa H, Roe AW (2010) A motion direction map in macaque V2. Neuron 68:1002-1013. CrossRef Medline

Martinez-Conde S, Macknik SL, Hubel DH (2000) Microsaccadic eye movements and firing of single cells in the striate cortex of macaque monkeys. Nat Neurosci 3:251-258. CrossRef Medline

McFarland JM, Bondy AG, Saunders RC, Cumming BG, Butts DA (2015) Saccadic modulation of stimulus processing in primary visual cortex. Nat Commun 6:8110. CrossRef Medline

McKee SP, Levi DM (1987) Dichoptic hyperacuity: the precision of nonius alignment. J Opt Soc Am A 4:1104-1108. CrossRef Medline

Mitchell JF, Sundberg KA, Reynolds JH (2009) Spatial attention decorrelates intrinsic activity fluctuations in macaque area V4. Neuron 63:879888. CrossRef Medline

Moreno-Bote R, Beck J, Kanitscheider I, Pitkow X, Latham P, Pouget A (2014) Information-limiting correlations. Nat Neurosci 17:1410-1417. CrossRef Medline

Neri P, Bridge H, Heeger DJ (2004) Stereoscopic processing of absolute and relative disparity in human visual cortex. J Neurophysiol 92:1880-1891. CrossRef Medline

Nienborg H, Bridge H, Parker AJ, Cumming BG (2004) Receptive field size in V1 neurons limits acuity for perceiving disparity modulation. J Neurosci 24:2065-2076. CrossRef Medline

Nienborg H, Bridge H, Parker AJ, Cumming BG (2005) Neuronal computation of disparity in $\mathrm{V} 1$ limits temporal resolution for detecting disparity modulation. J Neurosci 25:10207-10219. CrossRef Medline

Nienborg H, Cohen MR, Cumming BG (2012) Decision-related activity in sensory neurons: correlations among neurons and with behavior. Annu Rev Neurosci 35:463-483. CrossRef Medline

Nienborg H, Cumming B (2010) Correlations between the activity of sensory neurons and behavior: how much do they tell us about a neuron's causality? Curr Opin Neurobiol 20:376-381. CrossRef Medline

Nienborg H, Cumming BG (2006) Macaque V2 neurons, but not V1 neurons, show choice-related activity. J Neurosci 26:9567-9578. CrossRef Medline

Nienborg H, Cumming BG (2007) Psychophysically measured task strategy for disparity discrimination is reflected in V2 neurons. Nat Neurosci 10: 1608-1614. CrossRef Medline

Nienborg H, Cumming BG (2009) Decision-related activity in sensory neurons reflects more than a neuron's causal effect. Nature 459:89-92. CrossRef Medline

Nienborg H, Cumming BG (2014) Decision-related activity in sensory neurons may depend on the columnar architecture of cerebral cortex. J Neurosci 34:3579-3585. CrossRef Medline

Nienborg H, Roelfsema PR (2015) Belief states as a framework to explain extra-retinal influences in visual cortex. Curr Opin Neurobiol 32:45-52. CrossRef Medline

Pitkow X, Liu S, Angelaki DE, DeAngelis GC, Pouget A (2015) How can single sensory neurons predict behavior? Neuron 87:411-423. CrossRef Medline

Prince SJ, Pointon AD, Cumming BG, Parker AJ (2000) The precision of single neuron responses in cortical area V1 during stereoscopic depth judgments. J Neurosci 20:3387-3400. Medline
Purushothaman G, Bradley DC (2005) Neural population code for fine perceptual decisions in area MT. Nat Neurosci 8:99-106. CrossRef Medline

Rabinowitz NC, Goris RL, Cohen M, Simoncelli EP (2015) Attention stabilizes the shared gain of V4 populations. Elife 4:e08998. CrossRef Medline

Read JC, Cumming BG (2003) Measuring V1 receptive fields despite eye movements in awake monkeys. J Neurophysiol 90:946-960. CrossRef Medline

Roelfsema PR, Lamme VA, Spekreijse H (2004) Synchrony and covariation of firing rates in the primary visual cortex during contour grouping. Nat Neurosci 7:982-991. CrossRef Medline

Romo R, Hernández A, Zainos A, Lemus L, Brody CD (2002) Neuronal correlates of decision-making in secondary somatosensory cortex. Nat Neurosci 5:1217-1225. CrossRef Medline

Ruff DA, Cohen MR (2014a) Global cognitive factors modulate correlated response variability between V4 neurons. J Neurosci 34:16408-16416. CrossRef Medline

Ruff DA, Cohen MR (2014b) Attention can either increase or decrease spike count correlations in visual cortex. Nat Neurosci 17:1591-1597. CrossRef Medline

Shadlen MN, Britten KH, Newsome WT, Movshon JA (1996) A computational analysis of the relationship between neuronal and behavioral responses to visual motion. J Neurosci 16:1486-1510. Medline

Shiozaki HM, Tanabe S, Doi T, Fujita I (2012) Neural activity in cortical area V4 underlies fine disparity discrimination. J Neurosci 32:3830-3841. CrossRef Medline

Smolyanskaya A, Haefner RM, Lomber SG, Born RT (2015) A modalityspecific feedforward component of choice-related activity in MT. Neuron 87:208-219. CrossRef Medline

Thomas OM, Cumming BG, Parker AJ (2002) A specialization for relative disparity in V2. Nat Neurosci 5:472-478. CrossRef Medline

Uka T, DeAngelis GC (2003) Contribution of middle temporal area to coarse depth discrimination: comparison of neuronal and psychophysical sensitivity. J Neurosci 23:3515-3530. Medline

Uka T, DeAngelis GC (2004) Contribution of area MT to stereoscopic depth perception: choice-related response modulations reflect task strategy. Neuron 42:297-310. CrossRef Medline

Uka T, DeAngelis GC (2006) Linking neural representation to function in stereoscopic depth perception: roles of the middle temporal area in coarse versus fine disparity discrimination. J Neurosci 26:6791-6802. CrossRef Medline

Uka T, Tanabe S, Watanabe M, Fujita I (2005) Neural correlates of fine depth discrimination in monkey inferior temporal cortex. J Neurosci 25:10796-10802. CrossRef Medline

Umeda K, Tanabe S, Fujita I (2007) Representation of stereoscopic depth based on relative disparity in macaque area V4. J Neurophysiol 98:241252. CrossRef Medline

Westheimer G (1979) Cooperative neural processes involved in stereoscopic acuity. Exp Brain Res 36:585-597. Medline

Wimmer K, Compte A, Roxin A, Peixoto D, Renart A, de la Rocha J (2015) Sensory integration dynamics in a hierarchical network explains choice probabilities in cortical area MT. Nat Commun 6:6177. CrossRef Medline

Yu XJ, Dickman JD, DeAngelis GC, Angelaki DE (2015) Neuronal thresholds and choice-related activity of otolith afferent fibers during heading perception. Proc Natl Acad Sci U S A 112:6467-6472. CrossRef Medline 